\title{
Anabases
}

ANABASES Traditions et réceptions de l'Antiquité

$4 \mid 2006$

Varia

\section{François HARTOG, Évidence de l'histoire. Ce que voient les historiens}

\author{
Lucie Zoppi
}

\section{OpenEdition}

\section{Journals}

Édition électronique

URL : http://journals.openedition.org/anabases/2865

DOI : 10.4000/anabases.2865

ISSN : 2256-9421

\section{Éditeur}

E.R.A.S.M.E.

\section{Édition imprimée}

Date de publication : 1 octobre 2006

Pagination : 309-311

ISSN : 1774-4296

\section{Référence électronique}

Lucie Zoppi, «François Hartog, Évidence de l'histoire. Ce que voient les historiens », Anabases [En ligne],

4 | 2006, mis en ligne le 01 mai 2012, consulté le 22 septembre 2020. URL : http://

journals.openedition.org/anabases/2865; DOI : https://doi.org/10.4000/anabases.2865

Ce document a été généré automatiquement le 22 septembre 2020.

(c) Anabases 


\title{
François HARTOG, Évidence de l'histoire. Ce que voient les historiens
}

\author{
Lucie Zoppi
}

\section{RÉFÉRENCE}

François HARTOG, Évidence de l'histoire. Ce que voient les historiens, Paris, Éditions EHESS, coll. « Cas de figure 5 », 2005, 285 p.

16 euros / ISBN 2-7132-2069-6.

1 Le nouvel ouvrage de F. Hartog, se situe dans la continuité de ses autres livres. Dans une démarche à la croisée de l'épistémologie, de l'historiographie et de l'anthropologie historique, l'ouvrage que propose F. Hartog est un parcours dans lequel il s'agit de «questionner l'évidence»(p.13). Écrire l'évidence de l'histoire, c'est tout d'abord affirmer que l'histoire « va de soi », mais c'est tout aussi bien « ouvrir un doute, laisser place à un point d'interrogation: est-ce si évident après tout? Et puis, de quelle histoire parle-t-on ? [...] Qu'implique un faire de l'histoire et, d'abord, de qui et de quoi dépend-il ? » (p. 11). Deux autres définitions de l'évidence, issues de l'evidentia latine de Cicéron et traduisant le substantif grec enargeia, sont celle du philosophe, qui dit «ce qu'il voit comme il le voit»(p.12), et celle de l'orateur, qui transforme l'auditeur en spectateur en créant « une illusion de présence » affectant l'auditeur comme s'il était réellement présent (p.12). Entre les deux, entre la science et la fiction, où situer l'histoire? Un dernier sens de l'évidence est celui de «signe, marque, preuve, témoignage ", un sens qui est totalement assimilé par l'histoire telle que nous la concevons aujourd'hui. Des Anciens aux Modernes, il était évident de situer l'histoire entre la science et la fiction, le visible et l'invisible. De même, la manière de faire de l'histoire tendait vers une quête de la vérité. Or, la conjoncture du $\mathrm{xx}^{\mathrm{e}}$ siècle, avec la place nouvelle du présent, particulièrement explicitée par F. Hartog dans Régimes d'historicité. Présentisme et expériences du temps (2003), semble mettre ces formes 
d'évidence en question : que signifie aujourd'hui être historien, en particulier lorsque l'évidence semble passée du côté de la mémoire et du patrimoine?

Dans la première partie intitulée Voir dans l'Antiquité, six chapitres se succèdent selon un ordre chronologique. Les trois premiers concernent directement la question du voir et du dire, les autres poursuivent l'enquête de la vue chez trois historiens : Thucydide, Polybe et Denys d'Halicarnasse. Dans ce voyage à travers le temps, F. Hartog observe les héritages et les ruptures entre les historiens et les différentes histoires qu'ils construisent, et, à travers cela, s'interroge sur la relation de l'historien au récit, sur son rapport à l'écriture, à la politique. Dans le troisième chapitre Voir et dire : la voie grecque de l'histoire ( $\mathrm{VI}^{e}-\mathrm{IV}^{e}$ siècle av. J.-C.), F. Hartog rappelle que Thucydide, en opposition à Hérodote, remplace le verbe historein par suggraphein, «mettre par écrit », et présente son récit comme un ktêma, un acquis pour toujours (p. 61). Son histoire n'est plus écrite pour vaincre l'effacement des traces, l'oubli et donc le temps; elle se veut un acquis pour toujours, pour servir à éclairer le présent des hommes de l'avenir. Avec le verbe suggraphein apparaît un lien nécessaire entre histoire et écriture, et F. Hartog déplace l'attention du lecteur vers la question du récit. Sur ce point, il rappelle les positions d'Aristote qui situe l'histoire du côté du particulier et du dire, en opposition à la poésie tragique, qui lui est supérieure, du côté du général et de la création de récits (p. 34). Polybe tentera de bouleverser cette hiérarchie en ajoutant que l'histoire est une tragédie vraie. L'histoire est-elle simplement le récit des choses faites, narratio rerum gestarum ? F. Hartog fait le lien entre les historiens et la politique, les affaires de la cité. Il remarque que Thucydide ou Polybe deviennent historiens lorsqu'ils se trouvent en exil, forcés de prendre un certain recul par rapport à leur sujet (p. 43). C'est par ces choix que se construit une "pensée historique occidentale» (p.35) avec ses "évidences ». Ainsi, dès l'Antiquité, se forme une réflexion sur "l'historien comme sujet écrivant ", notait Wilamowitz.

3 Cependant le thème majeur de cette première partie est celui de la vue, du visible ou encore de l'évidence du visible. Dans le premier chapitre intitulé Les premiers choix, l'aède, inspiré par la Muse, est défini comme un "voyant»: il voyait de la même manière l'œuvre des hommes et celle des dieux. La séparation entre visible et invisible se crée après l'épopée, lorsque la Muse s'est tue. Une nouvelle relation à la vue, celle de l'historien, est à établir. Or, l'historien n'ayant pas recours à la vision de la Muse, il lui faut user d'une sorte de substitut avec certaines limites : l'historia (p. 33). Dans Voir et dire: la voie grecque de l'histoire ( $\mathrm{VI}^{e}-\mathrm{IV}^{e}$ siècle av. J.-C.), l'historien Hérodote mène une enquête qui s'appuie à la fois sur l'ouïe et sur la vue, mais il est aussi celui qui voit ce que les autres ne voient pas (p.60). Dans le chapitre suivant, L'œil de Thucydide et l'histoire "véritable», F. Hartog insiste sur le fait que Thucydide introduit au cœur de son travail la notion d'autopsie, le fait de voir par soi-même au présent (p. 78). Ainsi, deux sens interviennent dans le travail de l'historien : la vue et l'ouïe. Or, parmi ces deux moyens de faire l'histoire, les historiens antiques apportent plus de crédit à la vue qu'à l'ouïe. En effet, l'ouie se situe généralement du côté du colportage, de ce à quoi l'on n'assiste pas. Cependant, l'ouïe n'est pas simplement ce que l'on entend, rappelle F. Hartog dans le chapitre cinq intitulé Voir depuis Rome. Polybe et la première histoire universelle (p. 98). Polybe, par exemple, met du côté de l'oreille le savoir livresque de Timée! Ainsi, la définition de l'histoire change en fonction de ce que l'historien met sous ces mots de vue et d'ouïe, et du rapport qu'il entretient avec eux. 
4 Les six chapitres qui suivent sont des réflexions sur les Évidences modernes. Cette seconde partie de l'ouvrage est consacrée à une sélection d'auteurs qui ont pensé l'histoire, du XIX $x^{e}$ siècle à nos jours. Ce sont tour à tour Augustin Thierry, Jules Michelet, Fustel de Coulanges et Claude Lévi-Strauss qui sont étudiés par F. Hartog. Cette partie reprend la problématique de l'évidence de l'histoire à travers «leurs reformulations modernes » (p. 14). En effet, pour ces auteurs, l'histoire est aussi une affaire de vue, comme en témoigne leur effort constant pour faire disparaître la frontière entre l'événement passé et le récit de ce même événement, entre l'invisible et le visible. Une place particulière est réservée à Michelet, ce grand voyageur au cœur des émotions historiques, dans le chapitre qui lui est consacré Michelet, la vie, l'histoire. L'historien, selon Michelet, est créateur de vie, il redonne vie et émotion aux événements du passé par la qualité de son récit (p.155). Reprenant des questions au cœur des problématiques actuelles, un chapitre entier est consacré au rapport entre le témoin et l'historien, immédiatement suivi par un questionnement sur la conjoncture actuelle intitulé Conjoncture fin de siècle : l'évidence de l'histoire. F. Hartog insiste sur les dangers des confusions entre l'historien, le témoin et le juge, ou encore, entre l'histoire, la mémoire et le patrimoine (p. 215). Enfin, le dernier mot est donné à Michel de Certeau dans un Épilogue. F. Hartog le présente comme un «homme du voyage» (p. 240) ou un « historien sans territoire » (p. 243). Il expose la manière dont Michel de Certeau faisait de l'histoire comme une invitation : «[...] Certeau, lui, est surtout attentif aux parcours, traces, sillages, aux passages d'un espace dans un autre, aux frontières (envisagées plus comme lieux de passage que comme limites ou arrêts) [...]. Plus que de ruptures et de basculements, il est soucieux des effritements, érosions, glissements, remplois, translations: il travaille sur l'entre-deux» (p.243). Comment ne pas être tenté par cette pensée toujours en mouvement?

5 L'ouvrage de F. Hartog se présente comme un voyage. À travers un questionnement autour de la vue et de l'évidence, l'auteur invite à une réflexion sur l'histoire et le rôle de l'historien. S'inscrivant dans la suite logique de Régimes d'historicité. Présentisme et expériences du temps, l'ouvrage Évidence de l'histoire. Ce que voient les historiens propose des hypothèses, des définitions, et, tout aussi utiles, des "contre-définitions ", du rôle de l'historien contemporain.

\section{AUTEURS}

\section{LUCIE ZOPPI}

Université Toulouse II - Le Mirail

lu.zoppi@laposte.net 